PROCEEDINGS OF THE

AMERICAN MATHEMATICAL SOCIETY

Volume 138, Number 7, July 2010, Pages 2537-2546

S 0002-9939(10)10285-8

Article electronically published on February 26, 2010

\title{
ESSENTIAL NORMS OF COMPOSITION OPERATORS BETWEEN BLOCH TYPE SPACES
}

\author{
RUHAN ZHAO
}

(Communicated by Nigel J. Kalton)

\begin{abstract}
For $\alpha>0$, the $\alpha$-Bloch space is the space of all analytic functions $f$ on the unit disk $D$ satisfying

$$
\|f\|_{B^{\alpha}}=\sup _{z \in D}\left|f^{\prime}(z)\right|\left(1-|z|^{2}\right)^{\alpha}<\infty .
$$

Let $\varphi$ be an analytic self-map of $D$. We show that for $0<\alpha, \beta<\infty$, the essential norm of the composition operator $C_{\varphi}$ mapping from $B^{\alpha}$ to $B^{\beta}$ can be given by the following formula:
\end{abstract}

$$
\left\|C_{\varphi}\right\|_{e}=\left(\frac{e}{2 \alpha}\right)^{\alpha} \limsup _{n \rightarrow \infty} n^{\alpha-1}\left\|\varphi^{n}\right\|_{B^{\beta}}
$$

\section{INTRODUCTION}

Let $D$ be the unit disk in the complex plane $\mathbb{C}$, and let $H(D)$ be the space of analytic functions on $D$. For an analytic self-map $\varphi$ of $D$, the composition operator $C_{\varphi}$ for $f \in H(D)$ is defined as

$$
C_{\varphi}(f)=f \circ \varphi .
$$

The study of composition operators started in the late 60 s and became fairly active after that. One of the reasons is that it provides connections between operator theory and complex analysis and helps us to gain a deeper understanding of both areas. For general references on the theory of composition operators, see the two books 2 and 9 .

Recently, there has been a lot of work on composition operators between Bloch type spaces. See, for example, [4, [5], [8] and [11. Recall that, for $0<\alpha<\infty$, an analytic function $f$ is said to be in the Bloch type space $B^{\alpha}$, or $\alpha$-Bloch space, if

$$
\|f\|_{B^{\alpha}}=\sup _{z \in D}\left|f^{\prime}(z)\right|\left(1-|z|^{2}\right)^{\alpha}<\infty .
$$

We also say that $f \in B_{0}^{\alpha}$, the little $\alpha$-Bloch space, if

$$
\lim _{|z| \rightarrow 1}\left|f^{\prime}(z)\right|\left(1-|z|^{2}\right)^{\alpha}=0 .
$$

Received by the editors July 16, 2009, and, in revised form, October 25, 2009, November 2, 2009, and November 11, 2009.

2000 Mathematics Subject Classification. Primary 47B33; Secondary $46 \mathrm{E} 15$.

Key words and phrases. Composition operators, essential norms, Block type spaces.

(C)2010 American Mathematical Society Reverts to public domain 28 years from publication 
The space $B^{\alpha}$ is a Banach space under the norm

$$
\left\||f \||_{B^{\alpha}}=|f(0)|+\sup _{z \in D}\left|f^{\prime}(z)\right|\left(1-|z|^{2}\right)^{\alpha},\right.
$$

and $B_{0}^{\alpha}$ is the closure of polynomials in $B^{\alpha}$. It is known that when $\alpha=1, B^{\alpha}=B$, the classical Bloch space; when $0<\alpha<1, B^{\alpha}=\operatorname{Lip}_{1-\alpha}$, the analytic Lipschitz space which contains analytic functions $f$ on $D$ satisfying

$$
|f(z)-f(w)| \leq C|z-w|^{1-\alpha}
$$

for some constant $C>0$ and all $z, w \in D$; when $\alpha>1, B^{\alpha}=H_{\alpha-1}^{\infty}$, the weighted Banach space of analytic functions that contains analytic functions $f$ on $D$ satisfying

$$
\sup _{z \in D}|f(z)|\left(1-|z|^{2}\right)^{\alpha-1}<\infty .
$$

We refer to 12 for the above facts.

Recall that the essential norm $\|T\|_{e}$ of a bounded operator $T$ between Banach spaces $X$ and $Y$ is defined as the distance from $T$ to the space of compact operators from $X$ to $Y$. The essential norms of composition operators between Bloch type spaces have been determined by several authors. The following result is known:

Theorem A. Let $0<\alpha, \beta<\infty$ and $\varphi$ be an analytic self-map of the unit disk $D$. Then the essential norm of the composition operator $C_{\varphi}: B^{\alpha} \rightarrow B^{\beta}$ is

$$
\left\|C_{\varphi}\right\|_{e}=\lim _{s \rightarrow 1} \sup _{|\varphi(z)|>s}\left|\varphi^{\prime}(z)\right| \frac{\left(1-|z|^{2}\right)^{\beta}}{\left(1-|\varphi(z)|^{2}\right)^{\alpha}} .
$$

The result was proved by Montes-Rodríguez in [6] for the case where $\alpha=\beta=1$, and in [7] for the case where $0<\alpha=\beta<\infty$. The general case was proved by Contreras and Hernandez-Díaz in [1]. When $0<\alpha \leq 1$, the result was also proved by MacCluer and the author in 3 .

Recently, Wulan, Zheng and Zhu obtained the following result in [10.

Theorem B. Let $\varphi$ be an analytic self-map of D. Then $C_{\varphi}$ is compact on the Bloch space $B$ if and only if

$$
\lim _{n \rightarrow \infty}\left\|\varphi^{n}\right\|_{B}=0
$$

Notice that here $\varphi^{n}$ means the $n$-th power of $\varphi$. A natural question that arises from this result is whether we can get an essential norm formula for composition operators on $B$ in terms of $\varphi^{n}$. The purpose of this paper is to solve this problem. Actually, we obtain an essential norm formula for composition operators from $B^{\alpha}$ to $B^{\beta}$ for any $0<\alpha, \beta<\infty$ in terms of $\varphi^{n}$. Here is our main result.

Theorem 1.1. Let $0<\alpha, \beta<\infty$. Let $\varphi$ be an analytic self-map of the unit disk $D$. Then the essential norm of the composition operator $C_{\varphi}: B^{\alpha} \rightarrow B^{\beta}$ is

$$
\left\|C_{\varphi}\right\|_{e}=\left(\frac{e}{2 \alpha}\right)^{\alpha} \limsup _{n \rightarrow \infty} n^{\alpha-1}\left\|\varphi^{n}\right\|_{B^{\beta}} .
$$

The paper is organized as follows. In section 2 we first give a new characterization of bounded composition operators from $B^{\alpha}$ to $B^{\beta}$ in terms of $\varphi^{n}$. In section 3 we prove the lower bound in Theorem 1.1, and in section 4 we prove the upper bound. 


\section{Boundedness}

In this section we give the following new characterization of bounded composition operators from $B^{\alpha}$ to $B^{\beta}$.

Theorem 2.1. Let $0<\alpha, \beta<\infty$. Let $\varphi$ be an analytic self-map of the unit disk $D$. Then $C_{\varphi}: B^{\alpha} \rightarrow B^{\beta}$ is bounded if and only if

$$
\sup _{n \in \mathbb{N}} n^{\alpha-1}\left\|\varphi^{n}\right\|_{B^{\beta}}<\infty .
$$

In order to prove this result, we need the following lemma.

Lemma 2.2. Let $\alpha>0, n \in \mathbb{N}$ and $0 \leq x \leq 1$. Let $H_{n, \alpha}(x)=x^{n-1}\left(1-x^{2}\right)^{\alpha}$. Then $H_{n, \alpha}$ has the following properties:

(i)

$$
\max _{0 \leq x \leq 1} H_{n, \alpha}(x)=H_{n, \alpha}\left(r_{n}\right)= \begin{cases}1, & n=1 \\ \left(\frac{2 \alpha}{n-1+2 \alpha}\right)^{\alpha}\left(\frac{n-1}{n-1+2 \alpha}\right)^{(n-1) / 2}, & n \geq 2\end{cases}
$$

where

$$
r_{n}= \begin{cases}0, & n=1 \\ \left(\frac{n-1}{n-1+2 \alpha}\right)^{1 / 2}, & n \geq 2 .\end{cases}
$$

(ii) For $n \geq 1, H_{n, \alpha}$ is increasing on $\left[0, r_{n}\right]$ and decreasing on $\left[r_{n}, 1\right]$.

(iii) For $n \geq 1, H_{n, \alpha}$ is decreasing on $\left[r_{n}, r_{n+1}\right]$, and so

$$
\min _{x \in\left[r_{n}, r_{n+1}\right]} H_{n, \alpha}(x)=H_{n, \alpha}\left(r_{n+1}\right)=\left(\frac{2 \alpha}{n+2 \alpha}\right)^{\alpha}\left(\frac{n}{n+2 \alpha}\right)^{(n-1) / 2} .
$$

Consequently,

$$
\lim _{n \rightarrow \infty} n^{\alpha} \min _{x \in\left[r_{n}, r_{n+1}\right]} H_{n, \alpha}(x)=\left(\frac{2 \alpha}{e}\right)^{\alpha}
$$

The proof is an easy exercise in calculus. We omit the details here.

Proof of Theorem 2.1. Let $C_{\varphi}: B^{\alpha} \rightarrow B^{\beta}$ be bounded. To show that (2.1) is true, we follow the lines of the proof for the lower bound in Theorem 2.1 of [6]. Let the integer $n \geq 2$. Consider the function $z^{n}$. By Lemma 2.2.

$$
\left\|z^{n}\right\|_{B^{\alpha}}=\max _{z \in D} n|z|^{n-1}\left(1-|z|^{2}\right)^{\alpha}=n\left(\frac{2 \alpha}{n-1+2 \alpha}\right)^{\alpha}\left(\frac{n-1}{n-1+2 \alpha}\right)^{(n-1) / 2},
$$

where the maximum is attained at any point on the circle with radius

$$
r_{n}=\left(\frac{n-1}{n-1+2 \alpha}\right)^{1 / 2} .
$$

Thus

$$
\lim _{n \rightarrow \infty} n^{\alpha-1}\left\|z^{n}\right\|_{B^{\alpha}}=\left(\frac{2 \alpha}{e}\right)^{\alpha}
$$


Hence, there is a constant $C>0$, independent of $n$, such that $\left\|z^{n}\right\|_{B^{\alpha}} \leq C n^{1-\alpha}$. Let $f_{n}(z)=z^{n} /\left\|z^{n}\right\|_{B^{\alpha}}$. Then $\|f\|_{B^{\alpha}}=\left\|f_{n}\right\|_{B^{\alpha}}=1$. Thus

$$
\infty>\left\|C_{\varphi}\right\| \geq\left\|C_{\varphi} f_{n}\right\|_{B^{\beta}}=\frac{\left\|\varphi^{n}\right\|_{B^{\beta}}}{\left\|z^{n}\right\|_{B^{\alpha}}} \geq \frac{1}{C} n^{\alpha-1}\left\|\varphi^{n}\right\|_{B^{\beta}} .
$$

Thus (2.1) is true.

Conversely, let (2.1) be true. Then obviously $\|\varphi\|_{B^{\beta}}<\infty$. If $\sup _{z \in D}|\varphi(z)|<1$, then there is a number $r$, with $0<r<1$, such that $\sup _{z \in D}|\varphi(z)|<r$. In this case we can easily check that $C_{\varphi}: B^{\alpha} \rightarrow B^{\beta}$ is bounded. In the following, we assume that $\sup _{z \in D}|\varphi(z)|=1$.

For any integer $n \geq 1$, let

$$
D_{n}=\left\{z \in D: r_{n} \leq|\varphi(z)| \leq r_{n+1}\right\},
$$

where $r_{n}$ is given by (2.2). Let $m$ be the smallest positive integer such that $D_{m} \neq \emptyset$. Since $\sup _{z \in D}|\varphi(z)|=1, D_{n}$ is not empty for every integer $n \geq m$, and $D=$ $\bigcup_{n=m}^{\infty} D_{n}$. By Lemma 2.2, for every $n \geq m$,

$\min _{z \in D_{n}} n^{\alpha}|\varphi(z)|^{n-1}\left(1-|\varphi(z)|^{2}\right)^{\alpha} \geq n^{\alpha} H_{n, \alpha}\left(r_{n+1}\right)=\left(\frac{2 \alpha n}{n+2 \alpha}\right)^{\alpha}\left(\frac{n}{n+2 \alpha}\right)^{(n-1) / 2}$,

where $H_{n, \alpha}(x)=x^{n-1}\left(1-x^{2}\right)^{\alpha}$. Thus

$$
\lim _{n \rightarrow \infty} \min _{z \in D_{n}} n^{\alpha}|\varphi(z)|^{n-1}\left(1-|\varphi(z)|^{2}\right)^{\alpha} \geq\left(\frac{2 \alpha}{e}\right)^{\alpha} .
$$

Therefore, there exists a constant $\delta>0$ such that, for any $n \geq m$,

$$
\min _{z \in D_{n}} n^{\alpha}|\varphi(z)|^{n-1}\left(1-|\varphi(z)|^{2}\right)^{\alpha} \geq \delta
$$

Let $f \in B^{\alpha}$. Then

$$
\begin{aligned}
& \left\|C_{\varphi} f\right\|_{B^{\beta}}=\sup _{z \in D}\left|f^{\prime}(\varphi(z)) \| \varphi^{\prime}(z)\right|\left(1-|z|^{2}\right)^{\beta} \\
& =\sup _{n \geq m} \sup _{z \in D_{n}}\left|f^{\prime}(\varphi(z)) \| \varphi^{\prime}(z)\right|\left(1-|z|^{2}\right)^{\beta} \\
& =\sup _{n \geq m} \sup _{z \in D_{n}} \frac{\left|f^{\prime}(\varphi(z))\right|\left|\varphi^{\prime}(z)\right|\left(1-|z|^{2}\right)^{\beta} n^{\alpha}|\varphi(z)|^{n-1}\left(1-|\varphi(z)|^{2}\right)^{\alpha}}{n^{\alpha}|\varphi(z)|^{n-1}\left(1-|\varphi(z)|^{2}\right)^{\alpha}} \\
& \leq \frac{1}{\delta} \sup _{n \geq m} \sup _{z \in D_{n}} n^{\alpha-1}\left|f^{\prime}(\varphi(z))\right|\left(1-|\varphi(z)|^{2}\right)^{\alpha}\left|\left(\varphi^{n}\right)^{\prime}(z)\right|\left(1-|z|^{2}\right)^{\beta} \\
& \leq \frac{1}{\delta}\|f\|_{B^{\alpha}} \sup _{n \geq 1} n^{\alpha-1}\left\|\varphi^{n}\right\|_{B^{\beta}} .
\end{aligned}
$$

Thus $C_{\varphi}$ is bounded from $B^{\alpha}$ to $B^{\beta}$. The proof is complete.

It is well-known that, as a simple consequence of the Schwartz-Pick lemma, $C_{\varphi}$ is bounded on the Bloch space $B$ for any analytic self-map $\varphi$ of $D$. Hence, if we let $\alpha=\beta=1$ in Theorem 2.1, we get the following corollary.

Corollary 2.3. For any analytic self-map $\varphi$ of the unit disk D,

$$
\sup _{n \in \mathbb{N}}\left\|\varphi^{n}\right\|_{B}<\infty
$$




\section{The LOWER ESTIMATE}

Proof of Theorem [1.1; The lower estimate. Let $n \in \mathbb{N}$. Consider the function $z^{n}$. By Lemma 2.2.

$$
\left\|z^{n}\right\|_{B^{\alpha}}=\max _{z \in D} n|z|^{n-1}\left(1-|z|^{2}\right)^{\alpha}=n\left(\frac{2 \alpha}{n-1+2 \alpha}\right)^{\alpha}\left(\frac{n-1}{n-1+2 \alpha}\right)^{(n-1) / 2},
$$

where the maximum is attained at any point on the circle with radius

$$
r_{n}=\left(\frac{n-1}{n-1+2 \alpha}\right)^{1 / 2} .
$$

Clearly,

$$
\lim _{n \rightarrow \infty} n^{\alpha-1}\left\|z^{n}\right\|_{B^{\alpha}}=\left(\frac{2 \alpha}{e}\right)^{\alpha}
$$

Let $f_{n}(z)=z^{n} /\left\|z^{n}\right\|_{B^{\alpha}}$. Then $\left\|f_{n}\right\|_{B^{\alpha}}=1$ and $f_{n} \rightarrow 0$ weakly in $B^{\alpha}$ (see [3]). Thus, if $K$ is any compact operator from $B^{\alpha}$ to $B^{\beta}$, then

$$
\lim _{n \rightarrow \infty}\left\|K f_{n}\right\|_{B^{\beta}}=0 .
$$

Hence,

$$
\left\|C_{\varphi}-K\right\| \geq \limsup _{n \rightarrow \infty}\left\|\left(C_{\varphi}-K\right) f_{n}\right\|_{B^{\beta}} \geq \limsup _{n \rightarrow \infty}\left\|C_{\varphi} f_{n}\right\|_{B^{\beta}} .
$$

Thus, for $C_{\varphi}: B^{\alpha} \rightarrow B^{\beta}$,

$$
\begin{aligned}
\left\|C_{\varphi}\right\|_{e} & \geq \limsup _{n \rightarrow \infty}\left\|C_{\varphi} f_{n}\right\|_{B^{\beta}}=\limsup _{n \rightarrow \infty} \frac{1}{\left\|z^{n}\right\|_{B^{\alpha}}}\left\|\varphi^{n}\right\|_{B^{\beta}} \\
& =\limsup _{n \rightarrow \infty} \frac{1}{n^{\alpha-1}\left\|z^{n}\right\|_{B^{\alpha}}} n^{\alpha-1}\left\|\varphi^{n}\right\|_{B^{\beta}} \\
& =\left(\frac{e}{2 \alpha}\right)^{\alpha} \limsup _{n \rightarrow \infty} n^{\alpha-1}\left\|\varphi^{n}\right\|_{B^{\beta}} .
\end{aligned}
$$

\section{The upper estimate}

We need several lemmas to obtain the upper bound in Theorem 1.1 .

For $r \in(0,1)$, let $K_{r} f(z)=f(r z)$. Then $K_{r}$ is a compact operator on the space $B^{\alpha}$ or $B_{0}^{\alpha}$ for any positive number $\alpha$, with $\left\|K_{r}\right\| \leq 1$. The following lemma is from [3].

Lemma 4.1. Let $0<\alpha<1$. Then there is a sequence $\left\{r_{k}\right\}$, with $0<r_{k}<1$ tending to 1 , such that the compact operator $L_{n}=\frac{1}{n} \sum_{k=1}^{n} K_{r_{k}}$ on $B_{0}^{\alpha}$ satisfies:

(i) For any $t \in[0,1), \lim _{n \rightarrow \infty} \sup _{\|f\|_{B^{\alpha}} \leq 1|z| \leq t} \sup _{|z| \leq t}\left|\left(\left(I-L_{n}\right) f\right)^{\prime}(z)\right|=0$.

(ii) $\lim _{n \rightarrow \infty} \sup _{\|f\|_{B^{\alpha}} \leq 1} \sup _{z \in D}\left|\left(I-L_{n}\right) f(z)\right|=0$.

(iii) $\limsup _{n \rightarrow \infty}\left\|I-L_{n}\right\| \leq 1$.

Furthermore, these statements hold as well for the sequence of biadjoints $L_{n}^{* *}$ on $B^{\alpha}$.

The analogue of Lemma 4.1 for the case $\alpha=1$ is next, which is also given in [3]. 
Lemma 4.2. There is a sequence $\left\{r_{k}\right\}$, with $0<r_{k}<1$ tending to 1 , such that the compact operator $L_{n}=\frac{1}{n} \sum_{k=1}^{n} K_{r_{k}}$ acting on $B_{0}$ satisfies:

(i) For any $t \in[0,1), \lim _{n \rightarrow \infty} \sup _{\|f\|_{B} \leq 1} \sup _{|z| \leq t}\left|\left(\left(I-L_{n}\right) f\right)^{\prime}(z)\right|=0$.

(iia) $\limsup _{n \rightarrow \infty} \sup _{\|f\|_{B} \leq 1} \sup _{|z|>s}\left|\left(I-L_{n}\right) f(z)\right|\left(\log \frac{1}{1-|z|^{2}}\right)^{-1} \leq 1$, for s sufficiently close to 1 , and

(iib) $\lim _{n \rightarrow \infty} \sup _{\|f\|_{B} \leq 1|z| \leq s} \sup _{\mid}\left|\left(I-L_{n}\right) f(z)\right|=0$, for the above $s$.

(iii) $\limsup _{n \rightarrow \infty}\left\|I-L_{n}\right\| \leq 1$.

Furthermore, the same is true for the sequence of biadjoints $L_{n}^{* *}$ on $B$.

For the case where $\alpha>1$, we have the following lemma.

Lemma 4.3. Let $\alpha>0$. Then there is a sequence $\left\{r_{k}\right\}$, with $0<r_{k}<1$ tending to 1 , such that the compact operator $L_{n}=\frac{1}{n} \sum_{k=1}^{n} K_{r_{k}}$ on $B_{0}^{\alpha}$ satisfies:

(i) For any $s \in[0,1), \lim _{n \rightarrow \infty} \sup _{\|f\|_{B^{\alpha}} \leq 1} \sup _{|z| \leq s}\left|\left(\left(I-L_{n}\right) f\right)^{\prime}(z)\right|=0$.

(ii) For any $t \in[0,1), \lim _{n \rightarrow \infty} \sup _{\|f\|_{B^{\alpha}} \leq 1} \sup _{|z| \leq t}\left|\left(I-L_{n}\right) f(z)\right|=0$.

(iii) $\limsup _{n \rightarrow \infty}\left\|I-L_{n}\right\| \leq 1$.

Furthermore, these statements hold as well for the sequence of biadjoints $L_{n}^{* *}$ on $B^{\alpha}$.

Proof. The proofs of (i) and (iii) and the statement about the biadjoints $L_{n}^{* *}$ are the same as the proof of Proposition 2.1 in [7, so we omit them. To prove (ii), notice that $\left(I-L_{n}\right) f(0)=0$ for all $n \in \mathbb{N}$, so by Exercise 11 on p. 75 of [12], we have

$$
\left(I-L_{n}\right) f(z)=\int_{D} \frac{\left(\left(I-L_{n}\right) f\right)^{\prime}(w)\left(1-|w|^{2}\right)^{1+\alpha}}{\bar{w}(1-\bar{z} w)^{2+\alpha}} d A(w) .
$$

Given $t \in[0,1)$ and any $\varepsilon>0$, we choose $s \in[0,1)$ sufficiently close to 1 such that

$$
\frac{1-s}{s(1-t)^{2+\alpha}}<\varepsilon \text {. }
$$

Hence

$$
\begin{aligned}
I_{1} & =\sup _{\|f\|_{B^{\alpha} \leq 1}|z| \leq t} \sup _{D \backslash D_{s}} \frac{\left|\left(\left(I-L_{n}\right) f\right)^{\prime}(w)\right|\left(1-|w|^{2}\right)^{1+\alpha}}{|w||1-\bar{z} w|^{2+\alpha}} d A(w) \\
& \leq \sup _{\|f\|_{B^{\alpha} \leq 1}}\left\|\left(I-L_{n}\right) f\right\|_{B^{\alpha}} \sup _{|z| \leq t} \int_{D \backslash D_{s}} \frac{1-|w|^{2}}{|w||1-\bar{z} w|^{2+\alpha}} d A(w) \\
& \leq \frac{1}{s(1-t)^{2+\alpha}} \sup _{|z| \leq t} \int_{D \backslash D_{s}}\left(1-|w|^{2}\right) d A(w) \\
& \leq \frac{1}{s(1-t)^{2+\alpha}}(1-s)<\varepsilon
\end{aligned}
$$

as $s$ is sufficiently close to 1 . Here for the second inequality we have used (iii). Fix this $s$. From (i), there exists $N>0$ such that for any integer $n>N$,

$$
\sup _{\|f\|_{B^{\alpha}} \leq 1|z| \leq s} \sup _{|z|}\left|\left(\left(I-L_{n}\right) f\right)^{\prime}(z)\right|<\varepsilon .
$$


For such $n$ we have

$$
\begin{aligned}
I_{2} & =\sup _{\|f\|_{B^{\alpha}} \leq 1|z| \leq t} \sup _{D_{s}} \frac{\left|\left(\left(I-L_{n}\right) f\right)^{\prime}(w)\right|\left(1-|w|^{2}\right)^{1+\alpha}}{|w||1-\bar{z} w|^{2+\alpha}} d A(w) \\
& \leq \varepsilon \sup _{|z| \leq t} \int_{D_{s}} \frac{\left(1-|w|^{2}\right)^{1+\alpha}}{|w||1-\bar{z} w|^{2+\alpha}} d A(w) \\
& \leq \frac{\varepsilon}{(1-t)^{2+\alpha}} \sup _{|z| \leq t} \int_{D_{s}} \frac{\left(1-|w|^{2}\right)^{1+\alpha}}{|w|} d A(w) \\
& <C \varepsilon
\end{aligned}
$$

for some positive constant $C$. From (4.1), upon combining the above inequalities, we get that for $n>N$,

$$
\begin{aligned}
& \sup _{\|f\|_{B^{\alpha}} \leq 1|z| \leq t} \sup _{|z|}\left|\left(I-L_{n}\right) f(z)\right| \\
& \quad \leq \sup _{\|f\|_{B^{\alpha}} \leq 1|z| \leq t} \sup _{D} \frac{\left|\left(\left(I-L_{n}\right) f\right)^{\prime}(w)\right|\left(1-|w|^{2}\right)^{1+\alpha}}{|w||1-\bar{z} w|^{2+\alpha}} d A(w) \\
& \quad \leq I_{1}+I_{2}<(1+C) \varepsilon .
\end{aligned}
$$

Thus

$$
\lim _{n \rightarrow \infty} \sup _{\|f\|_{B^{\alpha}} \leq 1} \sup _{|z| \leq t}\left|\left(I-L_{n}\right) f(z)\right|=0 .
$$

The proof is complete.

Proof of Theorem 1.1: The upper estimate. If $\limsup n^{\alpha-1}\left\|\varphi^{n}\right\|_{B^{\beta}}=\infty$, then the result is trivially true. Now suppose

$$
\limsup _{n \rightarrow \infty} n^{\alpha-1}\left\|\varphi^{n}\right\|_{B^{\beta}}<\infty .
$$

Then, by Theorem 2.1. $C_{\varphi}$ is a bounded operator from $B^{\alpha}$ to $B^{\beta}$ and, clearly, $\|\varphi\|_{B^{\beta}}<\infty$.

Notice that if $\sup _{z \in D}|\varphi(z)|<1$, then we can easily see that $C_{\varphi}: B^{\alpha} \rightarrow B^{\beta}$ is compact (see, for example, p. 129 of [2]) and that both sides of (1.1) are 0, so (1.1) is trivially true. Hence, in the following we assume that $\sup _{z \in D}|\varphi(z)|=1$.

Let $\left\{L_{n}\right\}$ be the sequence of operators given in Lemmas 4.1, 4.2 and 4.3. Since each $L_{n}$ is compact as an operator from $B^{\alpha}$ to $B^{\alpha}, C_{\varphi} L_{n}: B^{\alpha} \rightarrow B^{\beta}$ is also compact and we have

$$
\begin{aligned}
\left\|C_{\varphi}\right\|_{e} & \leq \limsup _{n \rightarrow \infty}\left\|C_{\varphi}-C_{\varphi} L_{n}\right\|=\limsup _{n \rightarrow \infty}\left\|C_{\varphi}\left(I-L_{n}\right)\right\| \\
& =\limsup _{n \rightarrow \infty} \sup _{\|f\|_{B^{\alpha}} \leq 1}\left\|C_{\varphi}\left(I-L_{n}\right) f\right\|_{B^{\beta}} .
\end{aligned}
$$

We bound this last expression from above by

$$
\begin{aligned}
& \limsup _{n \rightarrow \infty} \sup _{\|f\|_{B^{\alpha} \leq 1}}\left|\left(I-L_{n}\right) f(\varphi(0))\right| \\
& \quad+\limsup _{n \rightarrow \infty} \sup _{\|f\|_{B^{\alpha} \leq 1}} \sup _{z \in D}\left|\left(\left(I-L_{n}\right) f\right)^{\prime}(\varphi(z)) \| \varphi^{\prime}(z)\right|\left(1-|z|^{2}\right)^{\beta} .
\end{aligned}
$$

Lemma 4.1 (ii), Lemma 4.2 (iib) and Lemma 4.3 (ii) guarantee that

$$
\limsup _{n \rightarrow \infty} \sup _{\|f\|_{B^{\alpha}} \leq 1}\left|\left(I-L_{n}\right) f(\varphi(0))\right|=0 .
$$


Now we need only consider the term

$$
J=\sup _{\|f\|_{B^{\alpha} \leq 1}} \sup _{z \in D}\left|\left(\left(I-L_{n}\right) f\right)^{\prime}(\varphi(z))\right|\left|\varphi^{\prime}(z)\right|\left(1-|z|^{2}\right)^{\beta} .
$$

For any integer $n \geq 1$, let $r_{n}$ be given by (2.2), and let

$$
D_{n}=\left\{z \in D: r_{n} \leq|\varphi(z)| \leq r_{n+1}\right\} .
$$

Let $m$ be the smallest positive integer such that $D_{m} \neq \emptyset$. Since $\sup _{z \in D}|\varphi(z)|=1$, $D_{n}$ is not empty for every integer $n \geq m$, and $D=\bigcup_{n=m}^{\infty} D_{n}$. Now we divide $J$ into two parts:

$$
\begin{aligned}
J= & \sup _{\|f\|_{B^{\alpha}} \leq 1} \sup _{m \leq k \leq N-1} \sup _{z \in D_{k}}\left|\left(\left(I-L_{n}\right) f\right)^{\prime}(\varphi(z)) \| \varphi^{\prime}(z)\right|\left(1-|z|^{2}\right)^{\beta} \\
& \quad+\sup _{\|f\|_{B^{\alpha}} \leq 1} \sup _{k \geq N} \sup _{z \in D_{k}}\left|\left(\left(I-L_{n}\right) f\right)^{\prime}(\varphi(z)) \| \varphi^{\prime}(z)\right|\left(1-|z|^{2}\right)^{\beta} \\
= & J_{1}+J_{2} .
\end{aligned}
$$

Here $N$ is a positive integer determined as follows. Write the function under three supremum signs in $J_{2}$ as

$$
\frac{\left|\left(\left(I-L_{n}\right) f\right)^{\prime}(\varphi(z))\right|\left|\varphi^{\prime}(z)\right|\left(1-|z|^{2}\right)^{\beta} k^{\alpha}|\varphi(z)|^{k-1}\left(1-|\varphi(z)|^{2}\right)^{\alpha}}{k^{\alpha}|\varphi(z)|^{k-1}\left(1-|\varphi(z)|^{2}\right)^{\alpha}} .
$$

By Lemma 2.2, for $z \in D_{k}$,

$$
k^{\alpha}|\varphi(z)|^{k-1}\left(1-|\varphi(z)|^{2}\right)^{\alpha} \geq\left(\frac{2 \alpha k}{k+2 \alpha}\right)^{\alpha}\left(\frac{k}{k+2 \alpha}\right)^{(k-1) / 2} .
$$

It is easy to see that

$$
\lim _{k \rightarrow \infty}\left[\left(\frac{2 \alpha k}{k+2 \alpha}\right)^{\alpha}\left(\frac{k}{k+2 \alpha}\right)^{(k-1) / 2}\right]^{-1}=\left(\frac{e}{2 \alpha}\right)^{\alpha} .
$$

Hence, for any $\varepsilon>0$, we can choose $N>m+1$ large enough such that for any $k \geq N$,

$$
\left[\left(\frac{2 \alpha k}{k+2 \alpha}\right)^{\alpha}\left(\frac{k}{k+2 \alpha}\right)^{(k-1) / 2}\right]^{-1}<\left(\frac{e}{2 \alpha}\right)^{\alpha}+\varepsilon
$$

For such $N$ we have

$$
\begin{aligned}
J_{2} & \leq\left[\left(\frac{e}{2 \alpha}\right)^{\alpha}+\varepsilon\right] \sup _{\|f\|_{B^{\alpha} \leq 1}}\left\|\left(I-L_{n}\right) f\right\|_{B^{\alpha}} \sup _{k \geq N} \sup _{z \in D_{k}} k^{\alpha-1}\left|\left(\varphi^{k}\right)^{\prime}(z)\right|\left(1-|z|^{2}\right)^{\beta} \\
& \leq\left[\left(\frac{e}{2 \alpha}\right)^{\alpha}+\varepsilon\right]\left\|I-L_{n}\right\| \sup _{k \geq N} k^{\alpha-1}\left\|\varphi^{k}\right\|_{B^{\beta}} .
\end{aligned}
$$

Thus by (iii) of Lemmas $4.1,4.2$ and 4.3 ,

$$
\limsup _{n \rightarrow \infty} J_{2} \leq\left[\left(\frac{e}{2 \alpha}\right)^{\alpha}+\varepsilon\right] \sup _{k \geq N} k^{\alpha-1}\left\|\varphi^{k}\right\|_{B^{\beta}}
$$

For $J_{1}$, by (i) of Lemmas 4.1, 4.2 and 4.3, we have

$$
\limsup _{n \rightarrow \infty} J_{1}=\limsup _{n \rightarrow \infty} \sup _{\|f\|_{B^{\alpha}} \leq 1 r_{m} \leq|\varphi(z)| \leq r_{N-1}}\left|\left(\left(I-L_{n}\right) f\right)^{\prime}(\varphi(z))\right|=0 .
$$

Hence, for any $N$ sufficiently large we have

$$
\left\|C_{\varphi}\right\|_{e} \leq \limsup _{n \rightarrow \infty} J_{2} \leq\left[\left(\frac{e}{2 \alpha}\right)^{\alpha}+\varepsilon\right] \sup _{k \geq N} k^{\alpha-1}\left\|\varphi^{k}\right\|_{B^{\beta}} .
$$


Hence

$$
\left\|C_{\varphi}\right\|_{e} \leq\left[\left(\frac{e}{2 \alpha}\right)^{\alpha}+\varepsilon\right] \limsup _{k \rightarrow \infty} k^{\alpha-1}\left\|\varphi^{k}\right\|_{B^{\beta}} .
$$

Since $\varepsilon$ is an arbitrary positive number, we have

$$
\left\|C_{\varphi}\right\|_{e} \leq\left(\frac{e}{2 \alpha}\right)^{\alpha} \limsup _{k \rightarrow \infty} k^{\alpha-1}\left\|\varphi^{k}\right\|_{B^{\beta}} .
$$

The proof is complete.

By (2.3) we know that

$$
\lim _{n \rightarrow \infty} n^{\alpha-1}\left\|z^{n}\right\|_{B^{\alpha}}=\left(\frac{2 \alpha}{e}\right)^{\alpha} .
$$

Combining this equation and Theorem 1.1, we immediately obtain the following corollary.

Corollary 4.4. Let $0<\alpha, \beta<\infty$. Let $\varphi$ be an analytic self-map of the unit disk $D$. Then the essential norm of the composition operator $C_{\varphi}: B^{\alpha} \rightarrow B^{\beta}$ is

$$
\left\|C_{\varphi}\right\|_{e}=\limsup _{n \rightarrow \infty} \frac{\left\|\varphi^{n}\right\|_{B^{\beta}}}{\left\|z^{n}\right\|_{B^{\alpha}}}=\limsup _{n \rightarrow \infty} \frac{\left\|C_{\varphi}\left(z^{n}\right)\right\|_{B^{\beta}}}{\left\|z^{n}\right\|_{B^{\alpha}}} .
$$

The following result is also an immediate consequence of Theorem 1.1 and Corollary 4.4 .

Corollary 4.5. Let $0<\alpha, \beta<\infty$. Let $\varphi$ be an analytic self-map of the unit disk $D$. Then the following conditions are equivalent:

(i) $C_{\varphi}$ is compact from $B^{\alpha}$ to $B^{\beta}$.

(ii) $\limsup _{n \rightarrow \infty} n^{\alpha-1}\left\|\varphi^{n}\right\|_{B^{\beta}}=0$.

(iii) $\limsup _{n \rightarrow \infty} \frac{\left\|\varphi^{n}\right\|_{B^{\beta}}}{\left\|z^{n}\right\|_{B^{\alpha}}}=0$.

Corollary 4.4 raises a natural question: For what other spaces $X$ and $Y$ of analytic functions on $D$ is the essential norm of the composition operator $C_{\varphi}$ from $X$ to $Y$ given by

$$
\left\|C_{\varphi}\right\|_{e}=\limsup _{n \rightarrow \infty} \frac{\left\|C_{\varphi}\left(z^{n}\right)\right\|_{Y}}{\left\|z^{n}\right\|_{X}} ?
$$

\section{REFERENCES}

1. M. Contreras and A. Hernandez-Díaz, Weighted composition operators in weighted Banach spaces of analytic functions, J. Australian Math. Soc., 69 (2000), 41-60. MR:1767392 (2001j:47027)

2. C. Cowen and B. MacCluer, Composition Operators on Spaces of Analytic Functions, CRC Press, Boca Raton, FL, 1995. MR1397026 (97i:47056)

3. B. MacCluer and R. Zhao, Essential norms of weighted composition operators between Blochtype spaces, Rocky Mountain J. Math., 33 (2003), 1437-1458. MR2052498 (2005b:47048)

4. K. M. Madigan, Composition operators on analytic Lipschitz spaces, Proc. Amer. Math. Soc., 119 (1993), 465-473. MR.1152987 (93k:47043)

5. K. M. Madigan and A. Matheson, Compact composition operators on the Bloch space, Trans. Amer. Math. Soc., 347 (1995), 2679-2687. MR.1273508 (95i:47061)

6. A. Montes-Rodríguez, The essential norm of a composition operator on Bloch spaces, Pacific J. Math., 188 (1999), 339-351. MR.1684196 (2000d:47044)

7. A. Montes-Rodríguez, Weighted composition operators on weighted Banach spaces of analytic functions, J. London Math. Soc. (2), 61 (2000), 872-884. MR.1766111 (2001j:47026) 
8. R. C. Roan, Composition operators on a space of Lipschitz functions, Rocky Mountain J. Math., 10 (1980), 371-379. MR575309 (81g:30046)

9. J. H. Shapiro, Composition Operators and Classical Function Theory, Springer-Verlag, New York, 1993. MR.1237406 (94k:47049)

10. H. Wulan, D. Zheng and K. Zhu, Compact composition operators on BMOA and the Bloch space, Proc. Amer. Math. Soc., 137 (2009), 3861-3868. MR2529895

11. J. Xiao, Composition operators associated with Bloch-type spaces, Complex Variables Theory Appl., 46 (2001), 109-121. MR.1867261 (2002k:47056)

12. K. Zhu, Operator Theory in Function Spaces, Marcel Dekker, New York, 1990. MR.1074007 (92c:47031)

Department of Mathematics, The College at Brockport, State University of New York, BrockPort, New York 14420

E-mail address: rzhao@brockport.edu 